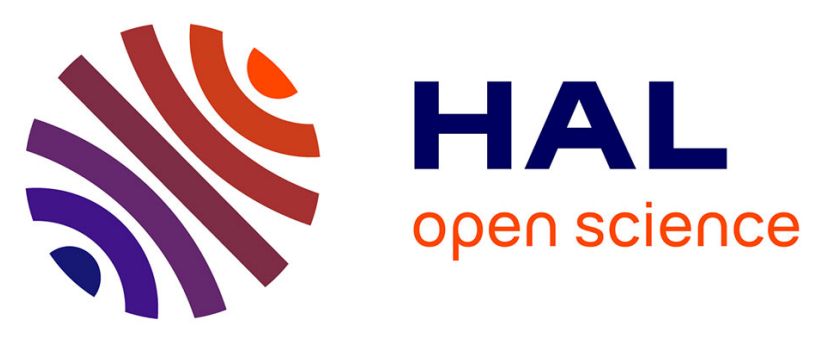

\title{
Lignes de fuite décoloniales
}

\author{
Elara Bertho, Anne Querrien
}

\section{To cite this version:}

Elara Bertho, Anne Querrien. Lignes de fuite décoloniales. Multitudes, 2021, n 84 (3), pp.52-56. 10.3917/mult.084.0052 . hal-03411630

\section{HAL Id: hal-03411630 \\ https://hal.science/hal-03411630}

Submitted on 18 Nov 2021

HAL is a multi-disciplinary open access archive for the deposit and dissemination of scientific research documents, whether they are published or not. The documents may come from teaching and research institutions in France or abroad, or from public or private research centers.
L'archive ouverte pluridisciplinaire HAL, est destinée au dépôt et à la diffusion de documents scientifiques de niveau recherche, publiés ou non, émanant des établissements d'enseignement et de recherche français ou étrangers, des laboratoires publics ou privés. 


\title{
Lignes de fuite décoloniales
}

\author{
Elara Bertho, Anne Querrien
}

Paru dans Multitudes $\underline{2021 / 3\left(n^{\circ} 84\right)}$, pages 52 à 56

https://doi.org/10.3917/mult.084.0052

Cette majeure est consacrée aux pensées contemporaines du décolonial, conçu non comme un temps historique (qui succéderait, comme le postcolonial, au temps de la colonisation), mais comme un processus continu et continué de déprise de la pensée marquée par la colonisation, comme un parcours de subjectivation. Aujourd'hui, aux quatre coins de la planète, des penseurs, des philosophes, des anthropologues, des littéraires proposent de nouvelles épistémologies. Elles et ils tentent de décentrer l'Europe. Avec quels récits, quels univers ? Avec quelles pensées du vivre-ensemble et du soin à la Terre ? Penser en dehors de la colonialité, oui, mais avec quels réseaux, quelles amitiés, quelles rencontres éclairantes?

Le constat est le suivant: la colonialité enserre nos schèmes de pensées, nos structures sociales globalisées, nos rêves, nos écosystèmes. Le décolonial désigne des formes plurielles de tentatives de se défaire du colonial. Si la décolonisation politique a donné lieu à ce qu'en Afrique on nomme les «Indépendances », la colonialité, au sens du sociologue péruvien Aníbal Quijano, demeure. Sous couvert d'un utopique rattrapage, elle est un système d'oppression, de sujétion des corps et des économies, d'extraction débridée des matières premières, de déstabilisation volontaire des communautés politiques qui tentent de se révolter. Sa force de frappe réside avant tout dans la diffusion à prétention universelle des savoirs, des imaginaires, des fantasmes élaborés dans le cadre de la colonie. L'efficacité pratique des savoirs occidentaux est aujourd'hui remise en cause par le maintien des disparités de conditions de vie. La prétention à proposer des visions universelles est battue en brèche par la prise en compte de la situation des savoirs, de leur caractère incarné. Dès lors la décolonialité a beaucoup à voir avec la notion de point de vue, de perspective, de regard. Tous termes qui désignent également le travail de l'écrivain ou de l'artiste.

\section{Des figures pour dire le trouble}

Rendre le contemporain intelligible. Décentrer le regard. Proposer des récits alternatifs. Figurer le possible. Que nous disent la littérature, les arts, les théoriciens du décolonial ? En quoi sont-ils des lieux de fabrication de la pensée décoloniale, en amont ou en marge des théoriciens de la décolonialité ? Que les écrivains se revendiquent ou non du décolonial importe peu. Leurs œuvres, par la mécanique même de la fabulation, peuvent penser le décolonial sans que ce soit une intention formulée par l'auteur. Une succession de figures complexes disent tout à la fois le symptôme du colonial (le manque, la zombification de l'être, la dépossession de soi-à-soi) et les multiples poussées de vie vers le décolonial (de luttes, de marronnages, de soin). Les figures qui suivent ne sont pas choisies comme des métaphores manichéennes qui incarneraient soit la sujétion soit la libération, puisque cette dualité ne rend pas compte de la complexité des rapports 
contemporains du colonial dans nos sociétés. Bien plutôt, ces figures sont des troubles : elles pointent un tremblement de la sujétion, une hypothèse en construction. Elles n'ont aucune prétention à être univoques, elles nomment des problématiques.

\section{Samba Diallo: il n'y a pas de dehors à l'aventure de la colonialité}

Dans L'aventure ambigüe, Cheik Hamidou Kane décrit la condition coloniale subalterne de manière paradigmatique : le jeune Samba Diallo est envoyé se former à l'école française, sur décision de sa tante, la Grande Royale, qui décrète que celui-ci doit apprendre des Blancs «l'art de vaincre sans avoir raison ». Ayant étudié la philosophie à Paris, il expérimente cette «aventure ambiguë » de dépossession de soi-à-soi, le clivage du colonisé.

Le roman, classique de la littérature africaine, ne rend pas uniquement compte de la fracture qu'ont vécue les élites formées dans les écoles occidentales, mais de la condition subalterne globale. C'est cette fracture que les théoriciens des années 1930-1940 ont tenté de soigner par les concepts de "Négritude», de "New Negro», concepts qui avaient vocation à redonner une dignité aux valeurs et cultures noires dans un contexte impérial. Cet homme scindé, c'est le symptôme de la colonialité invasive, venant remplacer de nouveaux rêves et de nouveaux fantasmes. Il n'y a pas de dehors à la condition subalterne : le symptôme de la colonialité, c'est d'incorporer le « rêve d'Autrui » jusqu'à la nausée.

L'aventure ambiguë, c'est le dialogue impossible, l'échec du langage à dire l'expérience de la dépossession, la déstructuration de soi par l'hégémonie blanche triomphante. Cheik Hamidou Kane écrit au moment où la Négritude, au Sénégal, est portée par Léopold Sédar Senghor, en partenariat étroit avec la maison d'édition Présence Africaine. La Négritude, de même que plus tard le postcolonial, interroge la hiérarchie de races, de valeurs, d'imaginaires, de territoires portées par la colonisation. Le décolonial découle des impasses de ces mouvements intellectuels à penser une alternative et de la recherche de lignes de fuite. L'aventure ambiguë, bien qu'écrite au moment « Négritude » de la pensée, intègre en réalité d'emblée des questions liées à la colonialité / décolonialité : l'incorporation de l'hégémonie à prétention universelle par le personnage principal, la somatisation du clivage, l'exigence de penser le dehors possible depuis la colonialité (qui est partout).

\section{Yaguine et Fodé : migrer pour « être comme vous "}

Les deux jeunes Guinéens sont décédés en 1999 dans un arrière-train d’un vol Conakry-Bruxelles. Dans une lettre emballée dans un sac plastique, ils disaient «aidez-nous à étudier pour être comme vous en Afrique ».

Yaguine et Fodé ont été mis en récit d'innombrables fois. Ils sont devenus un mythe, qui tout à la fois entre en écho et rend hommage à leur désir de réussir, puisqu'ils avaient pris soin d'emmener avec eux leurs carnets de résultats scolaires, excellents, dans l'espoir de poursuivre une scolarité en Europe. Les auteurs se sont emparés de ce qui ne pourrait être qu'un fait divers de plus dans la longue liste des migrants décédés lors de la traversée. Le dramaturge guinéen Hakim Bah leur donne la parole dans L'Avion va bientôt décoller puis À bout de sueurs, le plasticien Hassan Musa leur 
dédie Nage, Icare, dont le fond sinistre représente un Airbus, Marc Alexandre Oho Bambe (alias Capitaine Alexandre lorsqu'il est sur scène) reprend les deux noms dans Les Lumières d'Oujda - et l'on s'attend de fait à un destin tragique pour ces deux petits, ce qui ne manque pas d'arriver.

Ce que les textes littéraires disent de Yaguine et Fodé, c'est l'impasse de la fermeture des frontières qui appelle une pensée autre, décoloniale. Les processus migratoires sont induits par une économie de la prédation, rendant les pays de départ proprement invivables : c'est cette question de l'assujettissement des économies que pose le décolonial. La candeur de l'idée des deux enfants contraste avec la réalité de leur parcours. "Être comme vous », cette formule est le symptôme d'une marginalisation d'une partie de la planète qui n'a comme unique perspective que de rejoindre une inaccessible minorité.

\section{Sorcières coureuses de vents : utopies écoféministes}

Nnedi Okorafor, dans Kabu-Kabu, invente une série de personnages féminins, toutes «coureuses de vents", dans un cycle de nouvelles de science-fiction afroféministes. Ces femmes, marginalisées, exclues, traquées, sont dotées d'hypersensibilité et ont la capacité de courir parmi les vents. Héritières des sorcières, elles ont aussi la capacité de soigner par une connaissance particulière des plantes et de la forêt. Elles étaient respectées jusqu'aux « ères des étrangers », où les systèmes de valeurs ont été bouleversés et où les coureuses de vents ont été assimilées à de la sorcellerie, rendue incompréhensible et condamnable. Le lien avec la colonialité et le Plantationocène est établi clairement par Okorafor : les hommes ont oublié comment dialoguer avec la forêt, les arbres, les vents, les roches. La traque des coureuses de vents, leurs stratégies de fuite, leur reconversion en infirmières au sein de la guerre du Biafra : autant de trajectoires qui disent la lutte, le marronnage, la réappropriation de soi. Nnedi Okorafor, en investissant l'afrofuturisme, l'utopie écoféministe, ne prétend pas fournir des sorties concrètes de la colonialité : elle nomme, elle indexe une perte de lien. Ses personnages de coureuses de vents exposent des points fascinants de l'histoire : fascinants parce qu'elles renvoient à un élément très concret de la désacralisation des espaces et des lieux, de la suspicion portée sur les médecines féminines, fascinants aussi parce qu'elles passent par l'univers onirique pour résoudre et soigner la perte. De cette fascination naît une manière décoloniale de poser le problème : non pas établir un mode d'emploi de sortie de la colonialité, ce qui est un agenda impossible à tenir, mais proposer des agencements différents de ses éléments constituants. L'utopie est une boussole. Le trajet reste à faire, mais la question est posée par la fiction : c'est cela qu'apporte la coureuse de vents, une manière de poser la question.

Ces figures littéraires posent des problèmes qu'abordent les théoriciens et les militants du décolonial avec leurs propres coordonnées : la difficulté du dehors de la colonialité, le "rêve d'Autrui », les agencements écoféministes et les pensées du soin. Ce dossier sur la pensée décoloniale affronte la nécessité de complexifier le rapport centre/périphérie en proposant des récits pluriversels. La pensée décoloniale provoque chez ceux qui savent la reconnaître un décentrement de la vision qui correspond à ces nouvelles perspectives.

Ce dossier entend affronter la notion de « décolonial» dans toutes ses ambivalences et toutes ses promesses également. Les auteurs de cette majeure ne sont pas tous d'accord entre eux sur les acceptions du terme, et certains s'en écartent même significativement. C'est cet ensemble de regards, résolument hétérogène, que nous proposons ici. Il ne s'agit sans doute pas d'être pour ou contre le décolonial. Bien plutôt, nous souhaitons penser avec le décolonial, en ce sens que nous 
souhaitons montrer la grande richesse des questions que pose le décolonial aujourd'hui. C'est donc plus une manière de poser la question décoloniale qui nous intéresse ici.

Walter Mignolo, d'abord, rappelle la genèse du terme, issu des recherches d'Anibal Quijano. Le couple modernité/colonialité étant partout, la réponse décoloniale doit également émerger de partout, de manière pluriverselle. La décolonialité nomme précisément les énergies luttant pour une libération du savoir, partout, à l'échelle du globe. C'est cette énergétique de la lutte qui est également au cœur du texte de Jules Falquet: construit sur le même modèle archéologique que celui de Walter Mignolo, le texte de Jules Falquet rappelle les sources diverses du féminisme décolonial, ancrées dans la pensée d'Abya Yala, l'Amérique en langue locale, et les textes pionniers de Maria Lugones, qui a été la première à souligner les impensés de la question du sexe dans la théorisation décoloniale. Le schéma reproductif homme/femme est une imposition coloniale moderne: complexifier ce schéma, c'est penser l'intersectionnel dans le même mouvement que l'anticolonialisme, l'antiextractivisme et l'anticapitalisme. Toutes les luttes doivent être menées de front, sans hiérarchie entre elles. Les rapports sociaux de race, de genre, de sexe sont inextricablement mêlés. Ochy Curiel illustre cette contribution à partir de l'expérience du GLEFAS (Groupe latino-américain de formation et d'action féministe) : le décolonial n'est pas seulement une critique du savoir académique, il est profondément ancré dans des pratiques autogestionnaires de luttes, dans la rue, dans les associations, dans les organisations communautaires.

Ulyse Rabaté s'inscrit dans ce sillage décolonial, en proposant une analyse des textes de l'historien algérien Daho Djerbal et singulièrement de la notion possible de «contre-histoire » de l'Algérie, en mobilisant les notions de subalternité, de colonialité du savoir et du rapport de pouvoir induit dans l'écriture de l'histoire. Ces notions sont remobilisées et transférées pour l'étude des quartiers populaires en France : là où l'organisation de la gauche dite «traditionnelle » échoue à mobiliser les jeunes, quelles nouvelles énergies sont mises en œuvre pour susciter du politique ?

Joseph Tonda se démarque du terme «décolonial», en soulignant à quel point il est encore redevable de l'héritage de l'esclavage et de la colonisation. Pourtant, il conduit à des interrogations sur le décentrement des imaginaires. Il propose le terme d'« afrodystopie » pour décrire l'imposition du "rêve de l'Autre », le rêve du Blanc mâle hétérosexuel occidental, dans les imaginaires mondialisés. Mobilisant les images de Frankenstein, du zombie, tout en les croisant aux analyses anthropologiques de possession et des "maris de nuits", Tonda propose de repenser la notion de valeur dans l'économie de l'imaginaire globalisé.

Yves Citton et Emma Bigé se nourrissent de ces différentes discussions pour rappeler à quel point le décolonial est lié aux questions écologiques : l'ère du Plantationocène, cette économie globalisée inaugurée dans les colonies, fondée sur l'extractivisme et l'esclavagisme, met en danger le devenir même de la planète.

Si le décolonial devient la dernière idéologie activiste adoptée par les jeunes des pays nantis, il ne remplira pas ses promesses et continuera de rencontrer le silence de celles et ceux qu'il prétend représenter, s'inquiète Rey Chow devant cette nouvelle forme d'agitation universitaire. Giuseppe Cocco constate également une tendance des militants qui se réclament du décolonial à produire de la surenchère identitaire. Au Brésil cela se manifeste par un usage intempestif du paradigme anthropophagique, alors qu'historiquement l'anthropophagie fut une rencontre respectueuse de l'autre. Le féminisme décolonial porte pourtant l'espoir théorique et affectif d'un décentrement par rapport à la domination, et donc d'une libération, souligne Soumeya Mestiri. 
Apparu il y a une cinquantaine d'années en Amérique Latine et aux États-Unis dans les universités et dans la rue, le mouvement décolonial prend de nouvelles couleurs en Afrique où il cherche à redessiner le monde, essentiellement par la littérature et les arts plastiques comme Multitudes l'a déjà abordé dans le numéro 81 «Kinshasa star line » et dans le numéro 76 avec le suivi de quelques «Déplacements littéraires africains». Une recherche que nous entendons bien continuer. 